\title{
A VAR2CSA:CSP conjugate capable of inducing dual specificity antibody responses.
}

\author{
Matondo Sungwa ${ }^{1}$, Thrane Susan ${ }^{2}$, Janitzek Christoph Mikkel $^{2}$, Kavishe Reginald Adolph ${ }^{1}$, \\ Mwakalinga Steven Boniface ${ }^{1}$, Theander Thor Grundtvig ${ }^{2}$, Salanti Ali², \\ Nielsen Morten Agertoug$^{2}$, Sander Adam Frederik ${ }^{2}$.
}

1. Kilimanjaro Christian Medical University-College, and Kilimanjaro Clinical Research Institute, Moshi, Tanzania. 2. Centre for Medical Parasitology at Department of Immunology and Microbiology, Faculty of Health and Medical Sciences, University of Copenhagen and at Department of Infectious Diseases, Copenhagen University Hospital (Rigshospitalet), Denmark.

\begin{abstract}
:
Background: Vaccine antigens targeting specific P. falciparum parasite stages are under pre-clinical and clinical development. It seems plausible that vaccine with multiple specificities will offer higher protection. With this hypothesis, we exploited the SpyTag/SpyCatcher conjugation system to make a, post expression, dual antigen conjugate vaccine, comprising two clinically tested antigen candidates (CSP and VAR2CSA).

Methods: The DBL1x-DBL2x-ID2a region of VAR2CSA was genetically fused with SpyTag at N-terminus. The full-length CSP antigen was genetically fused to C-terminal SpyCatcher peptide. The covalent interaction between SpyTag/SpyCatcher enables the formation of DBL1x-DBL2x-ID2a:CSP conjugate vaccine. Immunogenicity and quality of antibody responses induced by the conjugate vaccine, as well as a control CSP-SpyCatcher vaccine, was tested in BALB/c mice.

Results: Serum samples obtained from mice immunized with the conjugate vaccine were able to recognize both untagged DBL1x-DBL2x-ID2a as well as CSP antigen. Moreover, the geometric mean anti-CSP antibody titer was 1.9-fold higher in serum (at day 35 and 55 post-first immunization) from mice immunized with the conjugate vaccine, as compared to mice receiving the control vaccine.

Conclusion: The data obtained in this study serves as proof-of-concept for the simultaneous induction of antibodies directed against individual antigen components in a dual stage anti-malaria vaccine.

Keywords: Malaria vaccine, Circumsporozoite protein, VAR2CSA, CSP-SpyCatcher, SpyTag-DBL1x-DBL2x-ID2a, bacterial superglue, DBL1x-DBL2x-ID2a:CSP conjugate.

DOI: https://dx.doi.org/10.4314/ahs.v17i2.11

Cite as: Sungwa M, Susan T, Mikkel JC, Adolph KR, Boniface MS, Grundtvig TT, Ali S, Agertong NM, Frederik SA. A VAR2CSA: CSP conjugate capable of inducing dual specificity antibody responses. Afri Health Sci. 2017;17(2): 373-381. https://dx.doi.org/10.4314/abs. $v 17 i 2.11$
\end{abstract}

\section{Introduction}

Plasmodium falciparum malaria remains a major public health problem as it continues to claim hundreds of thousands of lives globally each year. Pregnant women and children under 5 years of age, living in sub-Saharan Africa are the most affected groups ${ }^{1}$. Individuals acquire immunity as a function of malaria exposure ${ }^{2}$. Several measures have been taken by WHO to fight the disease, such as the use of long-lasting insecticide-treated nets (LLINs),

\section{Corresponding author: \\ Matondo Sungwa, Kilimanjaro Christian Medical University-College, P.O. Box 2236, Moshi, Tanzania. \\ Tel: +255 714433667, \\ Email: s.matondo@kcri.ac.tz}

indoor residual sprays (IRS) as well as anti-malarial drugs, which includes artemesinin-based combination therapies (ACTs $)^{1}$. However, none of these, or combinations hereof, have achieved elimination of the disease. A goal has been set by WHO to reduce malaria mortality by $90 \%$ in the year $2030^{1}$, and in the absence of an effective vaccine candidate it might be difficult to fulfill that goal on time. Development of effective anti-malaria vaccines has been hindered by the complexity of the parasite's life cycle as well as lack of complete knowledge concerning the interactions between the P.falciparum and host immune system, including mechanisms which regulate immune pathology in semi-immune individuals ${ }^{2}$. RTS,S/AS01 is the most advanced malaria vaccine candidate in terms of clinical development. The vaccine consists of a genetic fusion between the circumsporozoite protein (CSP) and a hepatitis B surface antigen embedded in lipid particles and

African Health Sciences Vol 17 Issue 2, June, 2017 
formulated with monophosphoryl lipid-A and Saponin. The fusion protein thus forms a virus-like particle (VLP) presenting a truncated form of CSP. The native CSP is expressed on sporozoites and thus the vaccine targets the pre-erythrocytic stage of the parasite. A recent large phase III clinical trial reported an efficacy of $37 \%$ protection in infants $(6-12 \text { weeks })^{3}$ and $47 \%$ in children $(5-15$ months $)^{4}$. However, widespread scale up of RTS,S/AS01 vaccination has not yet been finally endorsed by WHO partly due to the fast waning of protective anti-CSP antibodies ${ }^{1}$.

VAR2CSA is a candidate vaccine antigen for prevention of pregnancy-associated malaria (PAM). PAM is a special syndrome caused by sequestration of infected erythrocytes (IE) in the placenta, which can lead to poor pregnancy outcomes and death of both the mother and foetus ${ }^{5,6}$. The sequestration is mediated by the interaction between the human receptor condroitin sulphate A $(\mathrm{CSA})^{7}$ and VAR2CSA expressed by CSA binding parasite isolates ${ }^{8}$. Women in malaria endemic regions acquire protective circulating anti-VAR2CSA antibodies as a function of parity ${ }^{9}$, and protection is mediated by the ability of these antibodies to block the binding between CSA and VAR2CSA, thus preventing sequestration of IEs in the placenta ${ }^{10}$. Similarly, the VAR2CSA-based vaccine aims to target CSA-binding blood-stage parasites and prevent their accumulation in the placenta. However, VAR2CSA is a $350 \mathrm{kDa}$ antigen, which is made up of 6 Duffy binding-like domains (DBL) and 3 interdomain (ID) regions ${ }^{11}$. The size and complexity nature of VAR2CSA makes an expression of the full-length antigen a challenge for large scale expression, which is necessary for human clinical trial purposes. Therefore, it has been a strategy to design a vaccine based on only the CSA binding region of VAR2CSA ${ }^{12,13}$. Recently, two VAR2CSA candidate vaccines based on an N-terminal sub-fragment containing the minimal CSA binding region (ID1-DBL2x-ID2a) ${ }^{13}$ have gone into phase I trial in human volunteers.

It is reasonable to assume that a malaria vaccine targeting multiple stages of the parasite's life cycle will have increased efficacy. Such multistage vaccines are ideally constructed as genetic fusions to avoid multiple productions and stability testing. VAR2CSA is a complex protein with many cysteines forming disulfide bonds and it would require extensive experimental effort to produce a fusion construct to another complex malaria antigen.
Furthermore, fusion of two antigens might lead to dominant epitopes directing the response towards one of the antigens and thereby not inducing the desired multistage protective response. To examine if this would be the case, we constructed a dual antigen conjugate malaria vaccine targeting both the pre-erythrocytic stage of the parasite as well as the blood-stage of placental malaria infection. The two vaccine antigens (i.e. Full-length CSP and DBL1x-DBL2x-ID2a) were covalently linked, post-expression, by use of the (SpyTag/SpyCatcher) split-protein conjugation system (also can be termed as bacterial superglue ${ }^{14}$. This allows us to produce the antigens separately to ensure quality and folding. The dual antigen conjugate vaccine was tested in BALB/c mice in parallel with a control vaccine consisting of unfused CSP antigen. The titer of anti-DBL1x-DBL2x-ID2a and anti-CSP antibodies, respectively, was compared in mice immunized with the two vaccines to examine whether the co-valent conjugation of the two antigens had an effect on the immunogenicity of the CSP antigen component. Also the recognition of native VAR2CSA expression on IEs by the vaccine-induced anti-DBL1x-DBL2x-ID2a antibodies as well as anti-CSP antibodies from DBL1xDBL2x-ID2a:CSP conjugate and CSP-SpyCatcher vaccine respectively, was assessed by flow cytometry. The study serves as a proof of concept for simultaneous induction of antibodies against DBL1x-DBL2x-ID2a and $\mathrm{CSP}$ as a combined antigen. Future development of the vaccine would combine dual stage antigen linkage with VLP-display ${ }^{15}$.

\section{Methods \\ Design, expression and purification of VAR2C- SA-SpyTag antigen}

The DBL1x-DBL2x-ID2a region of VAR2CSA antigen from FCR3 genotype (Gene ID: 814364) was designed with C-terminally linked hexa-histidine purification tag (HIS), as well as SpyTag at the N-terminal ${ }^{15}$. A flexible linker made of serine/glycine was inserted between SpyTag and DBL1x-DBL2x-ID2a antigen. BamHI and NotI restriction site was introduced on SpyTag-DBL1xDBL2x-ID2a-HIS gene specifically at $\mathrm{N}$ - and C-terminus, respectively. The sequences were codon optimized and synthesized by GeneArt ${ }^{\circledR}$ Life Technologies (Germany), before cloning and expression in baculovirus transfected Spodoptera frugiperda (Sf9) insect cells as described previously by ${ }^{15,16}$. For recombinant expression, Sf9 cells were 
co-transfected with linear BakPak viral DNA (BD Biosciences) and pAcGP67A/Spy'Tag-DBL1x-DBL2x-ID2aHIS plasmid DNA using Lipofectamine 2000 Reagent (Invitrogen, 11668-019). Then transfected cells were subsequently incubated at $28^{\circ} \mathrm{C}$ for $3-5$ days, and high-tittered recombinant baculovirus was harvested from the supernatant and used for High-Five cells infection. The induction took place for 2 days, and was followed by centrifugation of cells (at $8000 \mathrm{~g}, 4^{\circ} \mathrm{C}, 10 \mathrm{~min}$ ), collection and filtering of the supernatant by $10 \mathrm{kDa}$ NMWC PES membranes $(0.45 \mu \mathrm{m})$ (56-4112-04; GE Healthcare). The supernatant was later diafiltrated six times on an ÄKTA cross-flow (GE Healthcare) and loaded on a 1-ml HisSelect column (H8286, Sigma-Aldrich) using an ÄKTA-express purification system (GE-Healthcare). Elution of the bound protein was performed using PBS buffer + $200 \mathrm{mM}$ imidazole (HisSelect), and lastly loaded directly onto a size exclusion chromatography HiLoad Superdex 200PG (28-9893-35, GE Healthcare). Vivaspin 20 columns were used to concentrate the monomeric peak (289323-60, GE Healthcare).

\section{Design, expression and purification of CSP-Spy- Catcher antigen}

Sequences coding a hexa-histidine purification tag was added at the 5 prime end of a construct coding a close to the full-length CSP sequence of amino acids 25-383 (in the native molecule amino acids 1-24 contains a N-terminal signal peptide and the sequence down-stream to 383 contains a C-terminal GPI-anchor sequence) derived from the 3D7 isolate (Gene ID: 814364), whereas DNA coding the SpyCatcher sequence was added at the 3 prime end $^{17}$. A flexible linker made of Glycine-Glycine-Serine amino acids was inserted between the CSP and SpyCatcher sequence. HIS-CSP-SpyCatcher gene was later modified to contain the BamHI and NotI restriction site at Nand C-terminus, respectively, and codon-optimized for synthesis (GeneArt ${ }^{\circledR}$ Life Technologies, Germany). The gene was subsequently expressed in Trichoplusia ni insect cells, as described previously by ${ }^{15,18}$.

\section{Conjugation of CSP-SpyCatcher and SpyTag- DBL1x-DBL2x-ID2a antigen}

CSP-SpyCatcher antigen (Fig. 1b) was conjugated to a Spy'Tag-DBL1x-DBL2x-ID2a antigen (Fig. 1a) by mixing of the two antigens in a molar ratio of 1:1 followed by overnight incubation at $4^{\circ} \mathrm{C}$. The reaction took place in a standard PBS buffer supplemented with $0.2 \%$ polysorbate 80 , with the PH value of 7.2. Mixing of the two constructs resulted in an efficient conjugation facilitated by the SpyTag/SpyCatcher interaction, which creates an isopeptide bond, and hence the formation of DBL1xDBL2x-ID2a:CSP conjugate (Fig. 1c). 
a

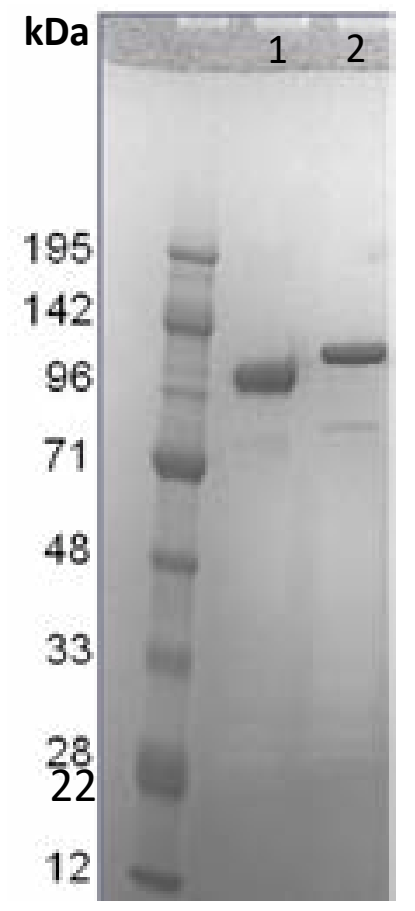

b

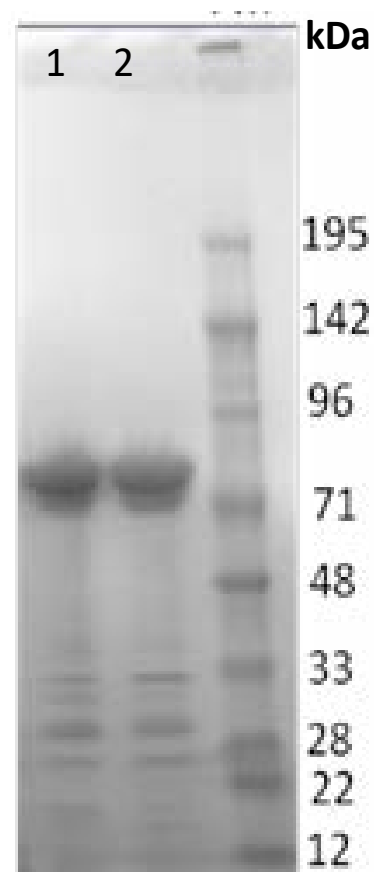

C

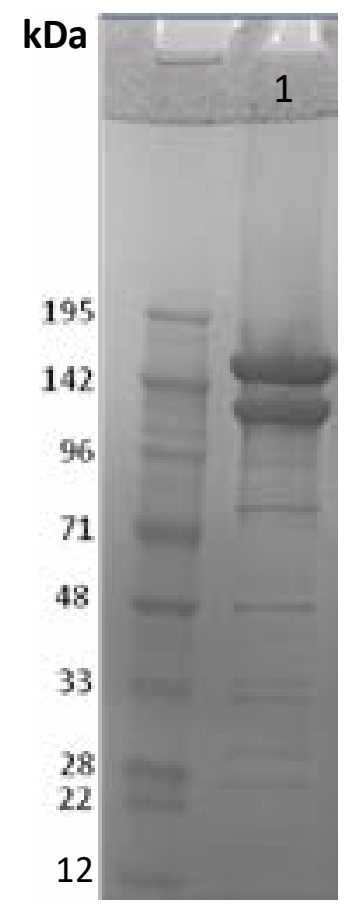

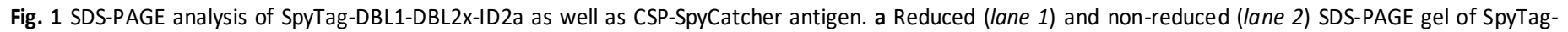



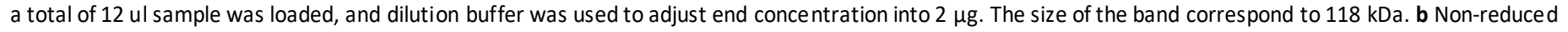

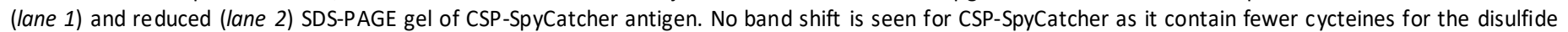

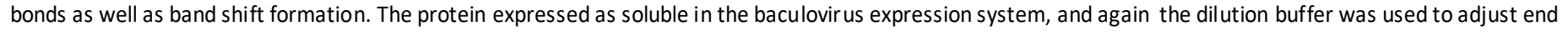

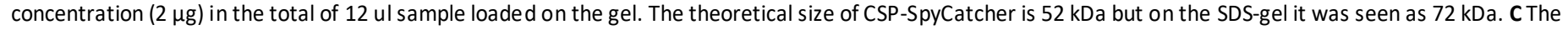

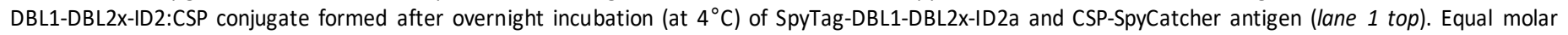

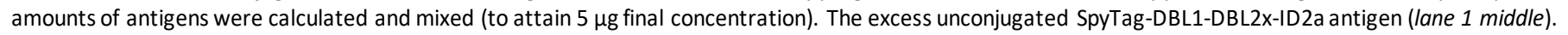

\section{Mouse immunization}

Female BALB/c mice (8 weeks old) (Taconic, Denmark) were immunized intramuscularly with $100 \mu$ of either DBL1x-DBL2x-ID2a:CSP or CSP-SpyCatcher vaccine (i.e. $5 \mu \mathrm{g}$ antigen dose) on days 0,21 and 42 . Both vaccines were formulated with Alum 1 hour prior to the immunizations. Mice sera were collected on days $0,14,35$, 49 and 55, and anti-DBL1x-DBL2x-ID2a and anti-CSP antibodies were measured by ELISA using untagged (i.e. no SpyTag or SpyCatcher) DBL1x-DBL2x-ID2a and CSP as capturing antigens.

\section{Antibody response measured by standard ELISA}

Standard enzyme-linked immunosorbent assay (ELISA) was used to measure mice serum Ig levels as described previously by ${ }^{15}$. In short, the recombinant DBL1xDBL2x-ID2a protein (without SpyTag) or full-length CSP (without SpyCatcher) was coated $(2 \mu \mathrm{g} / \mathrm{ml}$ in PBS) on a 96-well plate (Nunc MaxiSorp) by an overnight in- cubation at $4^{\circ} \mathrm{C}$. Plates were blocked with $1 \%$ BSA buffer at room temperature (RT) for 1 hour. 1:100 diluted mouse serum was then added in threefold dilutions to the wells, in triplicate, and was incubated for 1 hour at RT. Plates were washed three times in PBS with $0.05 \%$ tween 20 between individual steps. A 1:3000 dilution in blocking buffer of Horseradish peroxidase (HRP)-conjugated polyclonal goat anti-mouse IgG (Life Technologies, Denmark) was added and incubated for 1 hour. Color reactions were developed for $7 \mathrm{~min}$ by adding $\mathrm{O}$-phenylenediamine (OPD) (DAKO, Denmark) substrate. Termination of HRP enzymatic reaction was completed by adding 2.5 $\mathrm{M} \mathrm{H}_{2} \mathrm{SO}_{4}$ and the optical density (OD) was measured by an ELISA plate reader (VersaMax Molecular Devices) at $490 \mathrm{~nm}$. The arbitrary cutoff value of OD $490=0.2$ was used to determine the serum Ig endpoint titres. The geometric mean titres of anti-CSP antibodies were calculated both in mice sera derived from DBL1x-DBL2xID2a:CSP conjugate as well as CSP-SpyCatcher vaccine. 
Non-parametric, two tailed, Mann-Whitney Rank Sum Test statistical analysis was performed, and the P-value equivalent or less than 0.05 was regarded as statistically significant.

\section{P. falciparum cultures}

Parasite cultures were performed as described previously by $^{19}$. In short, maintenance of parasite culture was done in RPMI 1640 medium (Sigma Aldrich) mixed with 5\% hematocrit of human blood group $\mathrm{O}^{+}$blood. The RPMI medium was enhanced by the addition of $0.125 \mu \mathrm{g} / \mathrm{ml}$ Albumax II (Invitrogen), 2\% norm al human serum, $25 \mathrm{mmol} /$ liter sodium bicarbonate (Sigma Aldrich), and $0.125 \mu \mathrm{g} / \mathrm{ml}$ gentamicin. The selection of parasites for VAR2CSA expression was achieved by repeatedly panning of IEs on decorin (D8428, Sigma Aldrich) coated on the bottom of the culture flask. A day before the experiment $0.5 \mathrm{ml}$ of a sterile solution of $4 \mu \mathrm{g} / \mathrm{ml}$ decorin (D8428, Sigma Aldrich) was prepared in Dulbecco's PBS. The decorin solution was transferred to a culture flask by adding $0.5 \mathrm{ml}$ in one separate spot on the bottom of the flask, and the spot circumference was marked with a marker pen. It was followed by incubation at $4^{\circ} \mathrm{C}$ for overnight, whereas next day the flask was subsequently blocked by the addition of $5 \mathrm{ml}$ sterile $2 \%$ BSA (A7030, Sigma Aldrich) in RPMI medium, followed by incubation at $37^{\circ} \mathrm{C}$ for 2 hours. Afterwards the blocking medium was removed without allowing the spot to dry out, and $5 \mathrm{ml}$ of parasite culture medium was transferred to the decorin coated flask and gased. The IEs were allowed to adhere at $37^{\circ} \mathrm{C}$ for 1 hour at a gyrotable. Then after parasite culture was gently removed by washing with a heated $\left(\right.$ at $\left.37^{\circ} \mathrm{C}\right) \mathrm{RPMI}$ medium + gentamycin $(\mathrm{RBC}$ wash). The $\mathrm{RBC}$ washing was done by adding 3-5 $\mathrm{ml}$ of $\mathrm{RBC}$ wash (not directly on the spots), and very gently a small angle tilting of the flask was made to allow the slow wash away of unbound IEs. The medium with unbound erythrocytes was gently removed, and this step was repeated until erythrocytes were no longer visible in the medium supernatant. A light microscope was used for confirming the IEs-binding in the area of the spot, and not in the area outside the spots. $5 \mathrm{ml}$ medium as well as $200 \mu \mathrm{l}$ of blood $\left(5 \%\right.$ hematocrit of human blood group $\left.\mathrm{O}^{+}\right)$was transferred followed by gas. The cultures were screened for Mycoplasma and all tested negative. Also isolates were frequently genotyped for monitoring using MSP-2 as well as GLURP primers in a single PCR step.

\section{Flow cytometry}

The reactivity to native VAR2CSA expressed on FCR3-infected IEs was measured for antisera obtained from mice immunized with the dual antigen (DBL1x-DBL2xID2a:CSP) conjugate vaccine by flow-cytometry, as previously described $^{19}$. In brief, MACS purification of parasite lines (FCR3) suspended in PBS+2\%FBS was done to obtain late trophozoite and schizont stages after the exposure to a strong magnetic field. Aliquots of $50 \mu \mathrm{l}(2 \times 106$ IEs) of each parasite line in a 96-well plate was exposed to $50 \mu \mathrm{l}$ of mice serum and $100 \mu \mathrm{l}$ solution of 1:200 goat anti-mice FITC antibodies (Invitrogen, 629511) diluted in PBS $+2 \%$ FCS (PH 7.2), and $20 \mu \mathrm{l}$ of 1:50 (0.1 mg/ $\mathrm{ml}$ ) ethidium bromide. Data from 5000 infected cells was acquired from a $50 \mu \mathrm{l}$ of sample volume in a FC500 flow-cytometer (Beckman Coulter). Mean fluorescence intensities were subsequently analyzed using Winlist (Verity Software).

\section{Results}

\section{Immunogenicity of VAR2CSA:CSP and CSP-Spy-} Catcher vaccine

Antibodies in serum from mice immunized with both the dual antigen conjugate vaccine and the CSP-SpyCatcher vaccine reacted with untagged CSP antigen coated on ELISA plate (Fig. 2a), and full-bleed sera from mice immunized with the conjugate vaccine were also able to recognize untagged DBL1x-DBL2x-ID2a antigen (Fig. 2b). 
a



Day 55

b

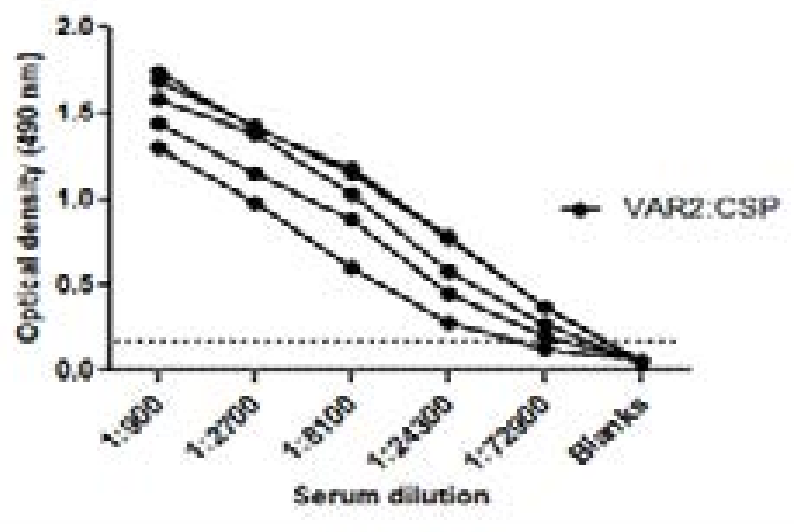

Fig. 2 ELISA showing the amount of anti-CSP IgG in sera from mice immunized with DBL 1x-DBL 2x-ID2a:CSP or CSP-SpyCatcher. A. Anti-CSP IgG titers in sera $(n=5)$ obtained from mice immunized (prime-boost-boost) with $5 \mu \mathrm{g}$ of either DBL1x-DBL2xID2a:CSP (filled circles) or CSP-SpyCatcher (open squares), using Aluminium hydroxide as extrinsic adjuvant. B. Anti-DBL1x-DBL2x-ID2a IgG in sera from mice $(\mathbf{n}=5)$ immunized (prime-boost-boost) with $5 \mu \mathrm{g}$ of the DBL1x-DBL2x-ID2a:CSP conjugate vaccine. The OD cut off value of 0.2 was used to determine endpoint titres.

The geometric mean endpoint titres of anti-CSP antibodies in the DBL1x-DBL2x-ID2a:CSP conjugate vaccine immunized group were slightly higher on days 35, 49 as well as 55 (Fig. 3), as compared to the CSP-SpyCatcher immunized group, but the difference was not statistical- ly significant for any of the days $(p=0.20, p=0.27$ and $\mathrm{p}=0.27$ respectively). Furthermore, 1.9-fold higher anti-CSP antibodies was observed both on day 35 and 55 in mice immunized with the conjugate vaccine as compared to the ones immunized with CSP-SpyCatcher vaccine, although the P-values were not statistically significant.

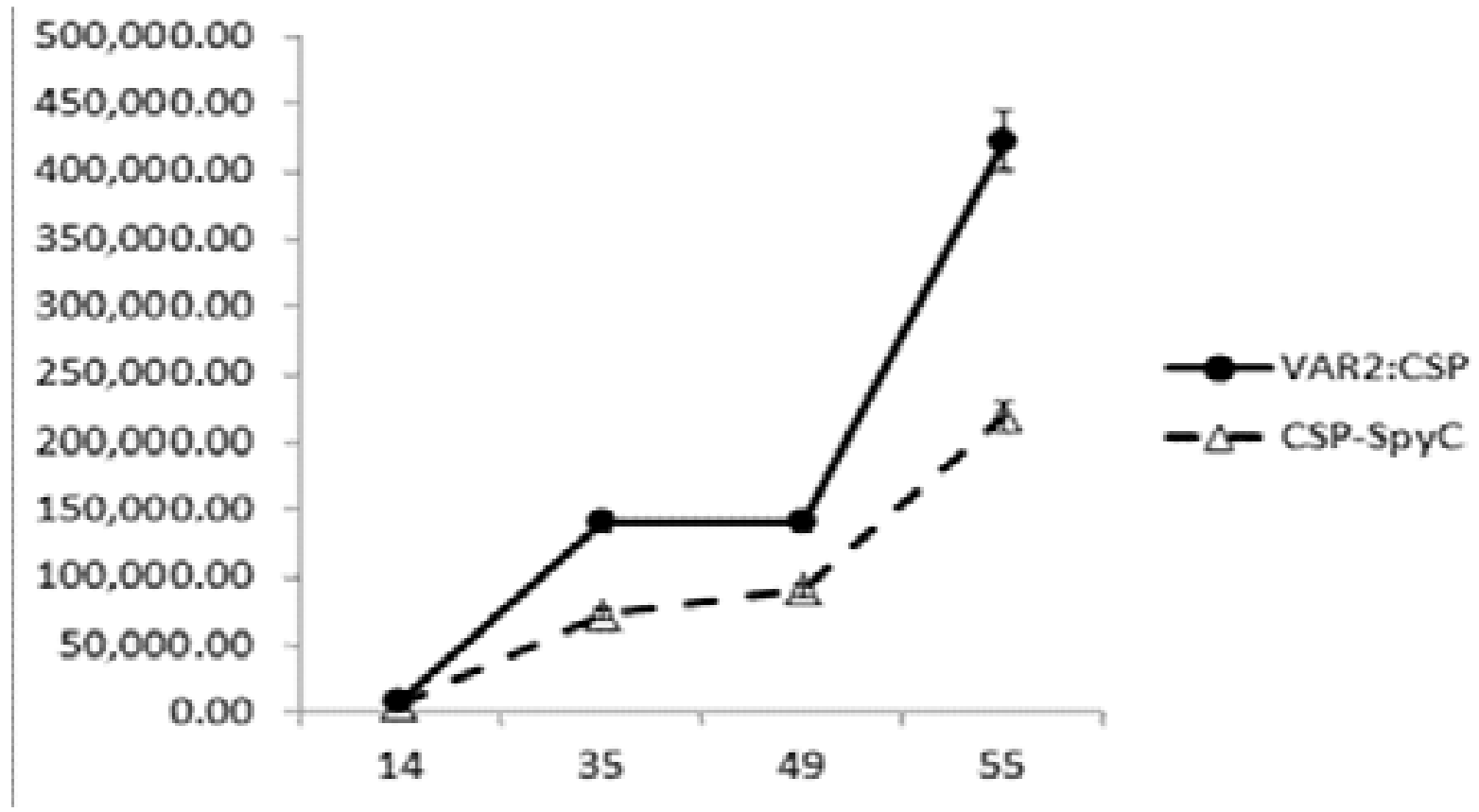

Fig. 3 Geometric mean titres. GMT of anti-CSP IgG antibodies in sera obtained from mice $(\mathrm{n}=5)$ immunized at days 14,35 , 49 and 55 with $5 \mu \mathrm{g}$ of DBL1x-DBL2x-ID2a:CSP (filled circles) or CSP-SpyCatcher (open triangles) formulated in aluminium hydroxide. Analyzed sera were obtained on days 14,35, 49 and 55, respectively. The geometric mean titer of sera obtained on day 35 and 55 from the DBL1x-DBL2x-ID2a:CSP vaccination group $(n=5)$ was more than 1.9-fold higher than in sera obtained at the same time points from the CSP-SpyCatcher vaccination group. P-values based on Mann-Whitney Rank sum test were $\mathrm{P}=0.20$ and $\mathrm{P}=0.27$, respectively. 
Recognition of native VAR2CSA expressed on the surface of the infected erythrocytes.

The FCR3 parasite line was selected for in-vitro CSA binding and VAR2CSA expression, and mature stage parasites were stained with sera from mice immunized with either the DBL1x-DBL2x-ID2a:CSP conjugate vaccine or CSP-SpyCatcher vaccine. All five mice sera raised from immunization with the DBL1x-DBL2x-ID2a:CSP conjugate vaccine gave rise to antibodies recognizing native VAR2CSA on the surface of IEs with a geometric mean above the level of CSP-SpyCatcher vaccine at the 1:10 dilution $(\mathrm{p}=0.0079)$ (Fig. 4).

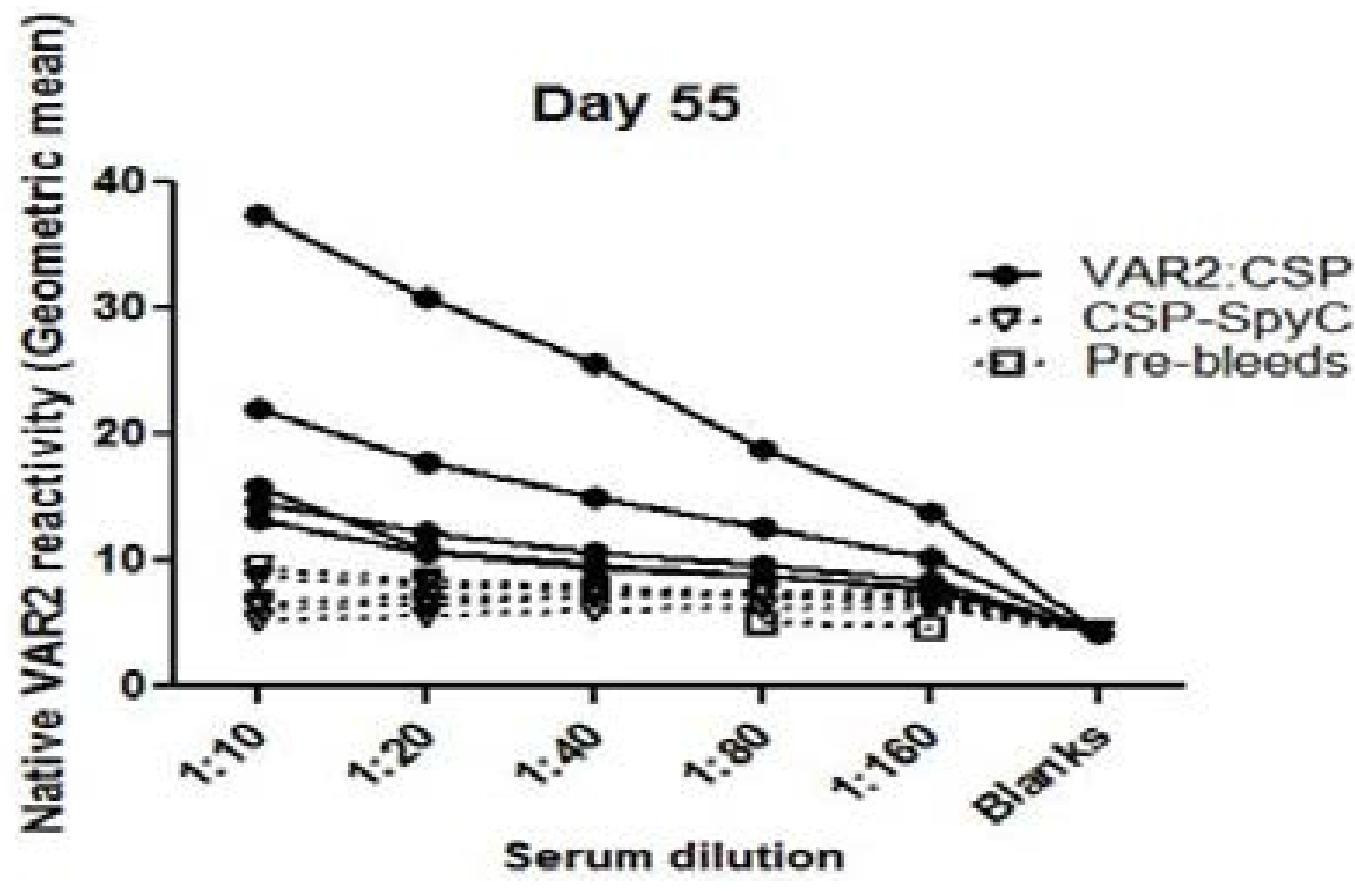

Fig. 4 Flow cytometry. Reactivity of sera obtained from mice $(n=5)$ immunized (prime-boost-boost) with $5 \mu \mathrm{g}$ of DBL1xDBL2x-ID2a: CSP (full circles) or CSP-SpyCatcher (open triangles) against native VAR2CSA (FCR3 genotype) expressed on the surface of infected red blood cells. Control sera (pre-bleeds) is shown as (open squares). The geometric mean recognition values for the DBL1x-DBL2x-ID2a:CSP group ranged between 7 and 37 whereas that of the CSP-SpyCatcher group ranged from 5 to 9 , and the difference between the groups was statistically significant $(\mathrm{p}=0.0079)$. In comparison, the negative control (pre-bleed) sera had the lowest geometric mean recognition values of less than 5.

\section{Discussion}

We have tested a dual stage malaria vaccine candidate that targets both the pre-erythrocytic liver stage as well as VAR2CSA-expressing blood-stage parasites infecting the placenta. The aim was to explore if fusion (or in this case, conjugation) would affect the specificity of the induced antibody responses towards the two subunit antigens. We found that the dual VAR2CSA:CSP conjugate vaccine induced antibodies that reacted with both recombinant VAR2CSA and native VAR2CSA expressed by the FCR3 parasite line. In addition, the conjugate vaccine gave rise to anti-CSP antibodies at a level 1.9-fold higher than unconjugated CSP on days 35 and 55, however the P-values were not statistically significant, which could be explained by an insufficient number of observations in our experiment. It is clear that it may not always be practical to produce a dual-antigen conjugate vaccines, but rather to include the individual antigens as separate entities in a single vaccine formulation. However, there may be cases where the immunogenicity of a vaccine antigen is improved by the covalent conjugation to another antigen. This is especially true for low-immunogenic antigens e.g.peptides, which are generally too small to elicit a sufficient immune response. Therefore, carrier proteins (e.g. bovine serum albumin, keyhole limpet hemocyanin or tetanus toxoid) that contain several epitopes have been used to stimulate T-helper cells, which subsequently facilitate the induction of the B-cell response. Traditionally, such protein-protein/protein-peptide conjugations have been done by chemical cross-linking chemistries, which generally require amino acid modifications (typically addition of a thiol group via a Cys side chain). Howev- 
er, the presence of multiple reactive functional groups may prevent site-specific conjugation and may lead to aggregates ${ }^{20}$. Therefore, the SpyTag/SpyCatcher conjugation system represents a novel easy-to-use tool to enable site-specific covalent conjugation of antigens and, in turn, enable comparison of immune responses raised against individual antigens prior to and post-conjugation. Besides, genetic fusion of the SpyTag/SpyCatcher rarely imposes a negative effect on the expression and folding of the antigen ${ }^{15}$ although in this study we did not examine whether native epitopes were retained in the CSP-SpyCatcher antigen.

In spite of many years of exposure women living in malaria endemic areas become susceptible to infection during pregnancy, indicating that naturally acquired protection against pre-erythrocytic parasite stages is not sufficient to protect these women. Vice versa, antibodies that target VAR2CSA are probably not protective in their own right rather synergising with semi-protective responses against the pre-erythrocytic and the merozoites stage, although such associations are inherently difficult to investigate. There are several candidate vaccines encompassing single antigens in on-going human clinical trials, including two VAR2CSA candidate vaccines based on the N-terminal sub-fragment containing the minimal binding region ${ }^{21}$. Clinical trials attempting to combine the pre-erythrocytic vaccines RTS/S-AS01 and ME-TRAP is likewise on-going $^{22}$. However, other pre-clinical studies combining vaccines against multiple parasite stages have not yet reached clinical testing to our knowledge $\mathrm{e}^{23,24}$. A dual or multi-specificity vaccine candidate could offer higher protection by decreasing both the number of parasites released from the liver and the number of parasites surviving in the blood. In addition, it appears increasingly important to induce responses that exceed what is acquired through natural infections ${ }^{22}$. As an example the RTS/S-AS01 vaccine induces many fold higher antibody levels than those found in serum of individuals living in endemic areas, however the problem of the vaccine appears to relate to its inability to induce sustained antibody responses. Novel vaccine technologies enabling presentation of complex antigens on the surface of the virus-like particles may be one way to increase the longevity of a response ${ }^{15,17}$. Hopefully, an endeavour to test a multi-component vaccine encompassing the current most promising vaccines against sporozoites, merozoites and infected erythrocytes will be possible in the future through uniting multiple different malaria vaccine centres.

\section{Acknowledgments}

The authors would like to thank Jens Hedelund Madsen, Elham Marjan and Nahla Chehabi for their technical support in cloning and expression as well as protein purification. Moreover, Anne Corfitz is thanked for technical support in flow cytometry and mice immunization.

\section{Authors' contributions}

SM and ST performed the experiments. SM, ST, CMJ, MAN, TGT, AS and AFS designed the experiments. SM, SBM, RAK and AFS were involved in writing the paper. All authors participated in the analysis of data. All authors read and approved the final manuscript.

\section{Ethical statement}

The study was approved by the Danish Animal Experiments Inspectorate. Approval number: 2013-15-293400902/BES.
Abbreviations
CSP - Circumsporozite Protein
HbsAg - Hepatitis B small surface antigen
PBS - Phosphate-buffered saline
HIS Hexa - histidine purification tag
Sf9 - Spodoptera frugiperda
DBL -Duffy Binding-like Domain
ID - Interdomain

\section{Conflict of interest}

For this project, we do not have any conflict of interest.

\section{References}

1. WHO. World Malaria Report. Geneva: World Health Organization; 2015. http://www.who.int/malaria/publications/world-malaria-report-2015/ report/en/. Accessed 18 Oct 2016.

2. Langhorne J, Ndungu FM, Sponaas AM, Marsh K. Immunity to malaria: more questions than answers. Nat Immunol. 2008; 9: 725-32. PubMed

3. Agnandji ST, Lell B, Fernandes JF, Abossolo BP, Methogo BGNO, Kabwende AL et al. A Phase 3 Trial of RTS,S/AS01 Malaria Vaccine in African Infants. N Engl $J$ Med 2012; 367(24): 2284-95. 
4. Agnandji ST, Lell B, Soulanoudjingar SS, Fernandes JF, Abossolo BP, Conzelmann C, et al. First results of phase 3 trial of RTS,S/AS01 malaria vaccine in African children. N Engl J Med 2011; 365(20): 1863-1875.

5. Brabin BJ, Romagosa C, Abdelgalil S, Mene'ndez C, Verhoeff FH, McGready R, et al. The Sick Placenta-The Role of Malaria. Placenta 2004; 25: 359-378.

6. Hviid L. The role of Plasmodium falciparum variant surface antigens in protective immunity and vaccine development. Human Vaccines 2010; 6(1): 84-89. PubMed

7. Fried M, Duffy PE. Adherence of Plasmodium falciparum to Chondroitin Sulfate A in the human placenta. Science 1996; 272(5267): 1502-1504. PubMed

8. Salanti A, Staalsoe T, Lavstsen T, Jensen ATR, Sowa MPK, Arnot DE, et al. Selective upregulation of a single distinctly structured var gene in chondroitin sulphate A-adhering Plasmodium falciparum involved in pregnancy associated malaria. Molecular Microbiology 2003; 49 (1): 179-191.

9. Salanti A, Dahlback M, Turner L, Nielsen MA, Barfod L, Magistrado P, et al. Evidence for the involvement of VAR2CSA in pregnancy-associated malaria. J Exp Med. 2004; 200(9): 1197-203. PubMed

10. Duffy PE, Fried M.Antibodies that inhibit Plasmodium falciparum adhesion to chondroitin sulfate $\mathrm{A}$ are associated with increased birth weight and the gestational age of Newborns. Infection and Immunity 2003; 71(11): 6620-6623.

11. Rask TS, Hansen DA, Theander TG, Pedersen AG, Lavstsen T. Plasmodium falciparum erythrocyte membrane protein 1 diversity in seven genomes - divide and conquer. PLoS Comput Biol 2010; 6(9): e1000933. PubMed

12. Dahlbäck M, Jørgensen LM, Nielsen MA, Clausen TM, Ditlev SB, Resende M, et al. The Chondroitin Sulfate A-binding Site of the VAR2CSA Protein involves multiple N-terminal Domains. J. Biol. Chem 2011; 286(18): 15908-15917.

13. Clausen TM, Christoffersen S, Dahlbäck M, Langkilde $\mathrm{AE}$, Jensen KE, Resende M, et al. Structural and functional insight into how the Plasmodium falciparum VAR2CSA protein mediates binding to chondroitin sulfate A in placental malaria. J. Biol. Chem 2012; 287(28): 23332-23345. 14. Zakeri B, Fierer JO, Celik E, Chittock EC, SchwarzLinek U, Moy VT, et al. Peptide tag forming a rapid covalent bond to a protein, through engineering a bacterial adhesin. Proc Natl Acad Sci USA. 2012; 109(12): 690-697. 15. Thrane S, Janitzek CM, Matondo S, Resende M, Gus- tavsson T, de Jongh WA, et al. Bacterial superglue enables easy development of efficient virus-like particle based vaccines. J. Nanobiotechnol. 2016; 14:30.

16. Wright KE, Hjerrild KA, Bartlett J, Douglas AD, Jin J, Brown RE, et al. Structure of malaria invasion protein RH5 with erythrocyte basigin and blocking antibodies. Nature. 2014; 7(7527): 427-430. PubMed

17. Janitzek CM, Matondo S, Thrane S, Nielsen MA, Kavishe $\mathrm{R}$, Mwakalinga SB, et al. Bacterial superglue generates a full length circumsporozoite protein virus like particle vaccine capable of inducing high and durable antibody responses. Malar J 2016; 15:545.

18. Khunrae P, Dahlbäck M, Nielsen MA, Andersen G, Ditlev SB, Resende M, et al. Full-length recombinant Plasmodium falciparum VAR2CSA binds specifically to CSPG and induces potent parasite adhesion-blocking antibodies. J Mol Biol. 2010; 397: 826- 34. PubMed

19. Nielsen MA, Pinto VV, Resende M, Dahlbäck M, Ditlev SB, Theander TG, et al. Induction of adhesion-inhibitory antibodies against placental Plasmodium falciparum parasites by using single domains of VAR2CSA. Infect Immun. 2009; 77(6): 2482-2487.

20. Lateef SS, Gupta S, Jayathilaka LP, Krishnanchettiar S, Huang Jin-S, Lee Bao-S. An improvedprotocol for coupling synthetic peptides to carrier proteins for antibody production using DMF to solubilize peptides. Journal of biomolecular Techniques. 2007; 18: 173-176.

21. Nielsen MA, Resende M, de Jongh WA, Ditlev SB, Mordmüller B, Houard S, et al. The influence of sub-unit composition and expression system on the functional antibody response in the development of a VAR2CSA based Plasmodium falciparum placental malaria vaccine. PLoS ONE. 2015; 10(9): e0135406. PubMed

22. Ewer KJ, Sierra-Davidson K, Salman AM, lllingworth JJ, Draper SJ, Biswasa S, et al. Progress with viral vectored malaria vaccines: A multi-stage approach involving "unnatural immunity". Vaccine 2015; 33(52): 7444-7451. PubMed

23. Spiegel H, Boes A, Kastilan R, Kapelski S, Edgue G, Beiss $\mathrm{V}$, et al. The stage-specific in vitro efficacy of a malaria antigen cocktail provides valuable insights into the development of effective multi-stage vaccines. Biotechnol J 2015; 10(10): 1651-1659. PubMed

24. Baldwin SL, Roeffen W, Singh SK, Tiendrebeogo RW, Christiansen M, Beebe E, et al. Synthetic TLR4 agonists enhance functional antibodies and CD4+ T-cell responses against the Plasmodium falciparum GMZ2.6C multi-stage vaccine antigen. Vaccine 2016; 34(19): 2207-2215. PubMed 